\title{
Acquired platinum resistance involves epithelial to mesenchymal transition through ubiquitin ligase FBX032 dysregulation
}

\author{
Nobuyuki Tanaka, ${ }^{1}$ Takeo Kosaka, ${ }^{1}$ Yasumasa Miyazaki, ${ }^{1}$ Shuji Mikami, ${ }^{2}$ Naoya Niwa, ${ }^{1}$ \\ Yutaro Otsuka, ${ }^{3}$ Yoji Andrew Minamishima, ${ }^{4}$ Ryuichi Mizuno, ${ }^{1}$ Eiji Kikuchi, ${ }^{1}$ Akira Miyajima, \\ Hisataka Sabe, ${ }^{3}$ Yasunori Okada, ${ }^{5}$ Per Uhlén, ${ }^{6}$ Makoto Suematsu, ${ }^{4}$ and Mototsugu Oya ${ }^{1}$ \\ 'Department of Urology, Keio University School of Medicine, Tokyo, Japan. 'Division of Diagnostic Pathology, Keio \\ University Hospital, Tokyo, Japan. ${ }^{3}$ Department of Molecular Biology, Hokkaido University Graduate School of Medicine, \\ Sapporo, Japan. ${ }^{4}$ Department of Biochemistry, Keio University School of Medicine, and Japan Agency for Science and \\ Technology (JST), Exploratory Research for Advanced Technology (ERATO), Suematsu Gas Biology Project, Tokyo, Japan. \\ ${ }^{5}$ Department of Pathology, Keio University School of Medicine, Tokyo, Japan. ${ }^{6}$ Department of Medical Biochemistry and \\ Biophysics, Karolinska Institutet, Stockholm, Sweden.
}

To identify the molecules involved in epithelial to mesenchymal transition (EMT) in urothelial carcinoma (UC) after acquisition of platinum resistance, here we examined the changes in global gene expression before and after platinum treatment. Four invasive UC cell lines, T24, 5637, and their corresponding sublines T24PR and 5637PR with acquired platinum resistance, were assessed by microarray, and the ubiquitin E3 ligase FBX032 was newly identified as a negative regulator of EMT in UC tumors after acquisition of platinum resistance. In vitro and in vivo studies showed an intimate relationship between FBX032 expression and EMT, demonstrating that FBX032 dysregulation in T24PR cells results in elevated expression of the mesenchymal molecules SNAIL and vimentin and decreased expression of the epithelial molecule E-cadherin. The association between FBX032 expression and EMT was further validated using clinical samples. Knockdown of MyoD expression, a specific target of FBXO32 polyubiquitination, revealed upregulation of E-cadherin expression and downregulation of SNAIL and vimentin expression in T24PR cells. Comparative genomic hybridization array analysis demonstrated loss of heterozygosity at 8q24.13 in T24PR cells, which harbors FBX032. Our findings suggest the importance of the association between EMT and ubiquitin-proteasome regulation when tumors develop acquired platinum resistance.

Authorship note: N. Tanaka and T. Kosaka contributed equally to this work.

Conflict of interest: Takeo Kosaka has received honoraria from Astellas and Sanofi. Ryuichi Mizuno has received honoraria from Novartis and Pfizer. Mototsugu Oya has received honoraria from Astellas, Bayer, Novartis, and Pfizer.

Submitted: July 23, 2015

Accepted: October 5, 2016

Published: November 3, 2016

Reference information:

JCI Insight. 2016;1(18):e83654

doi:10.1172/jci.insight.83654.

\section{Introduction}

Urothelial carcinoma (UC) is one of the most aggressive epithelial tumors, owing to early local/systemic dissemination $(1,2)$. Patients with metastatic disease are routinely treated with cisplatin-based (CDDPbased) chemotherapy, such as M-VAC (methotrexate, vinblastine, doxorubicin, CDDP) and/or GC (gemcitabine, CDDP) regimens. However, the vast majority of tumors develop to progressive disease (3), eventually acquiring platinum resistance.

Accumulating evidence suggests molecular and phenotypic association between chemoresistance and the acquisition of epithelial to mesenchymal transition (EMT) in cancer (4-11). The phenotypic hallmark of EMT is loss of E-cadherin, and several experimental models have documented a relationship between EMT and acquired resistance to chemotherapy (12-19). Originally, the process of EMT was observed during embryonic development (20), and a growing body of evidence has suggested that acquisition of EMT is also important for cancer progression and metastasis (21-23). Among the EMT-associated molecules, we have previously elucidated the impact of SNAIL, a member of a super family of zinc-finger transcriptional factors, on the determination of EMT characteristics and tumor invasion in patients with UC (24). However, with regard to the details by which acquired resistance to chemotherapy involves EMT in tumors, only scant evidence is currently available. 
A

T24 T24PR

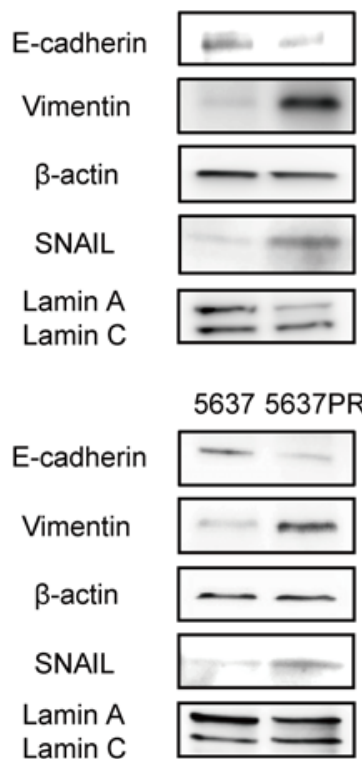

B

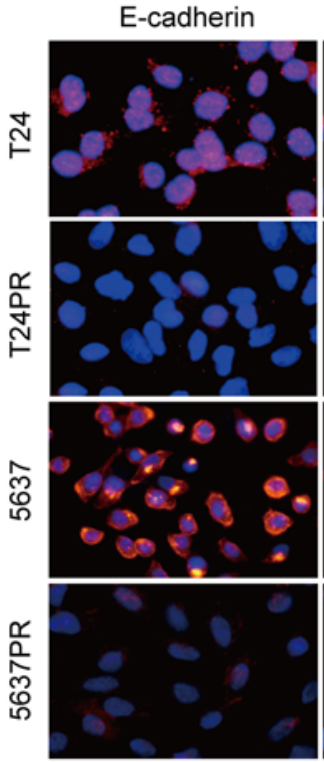

DAPI/E-cadherin

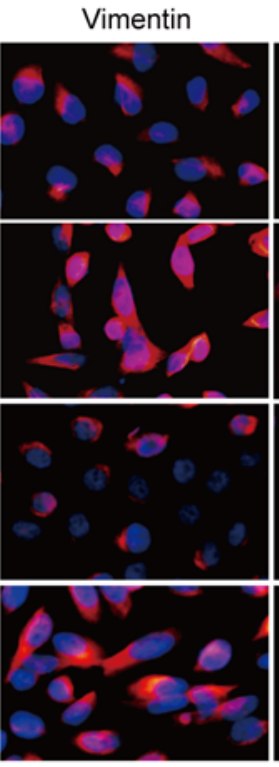

DAPINimentin

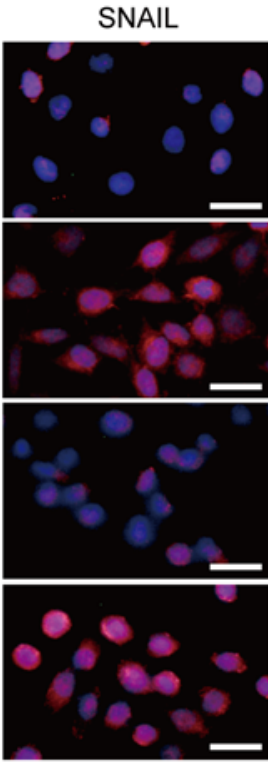

DAPI/SNAIL

\section{C}

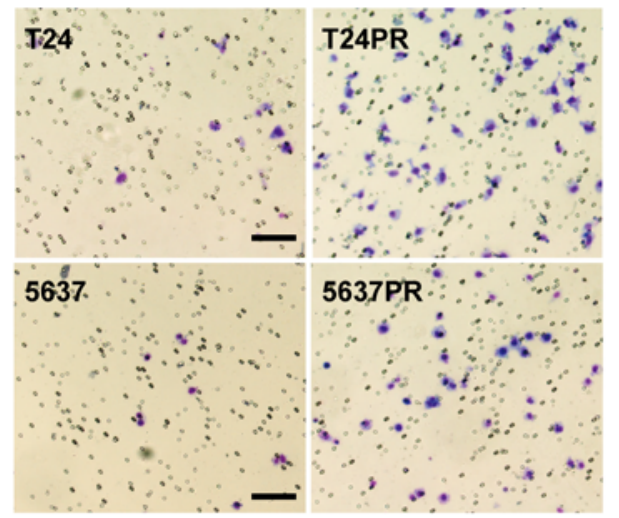

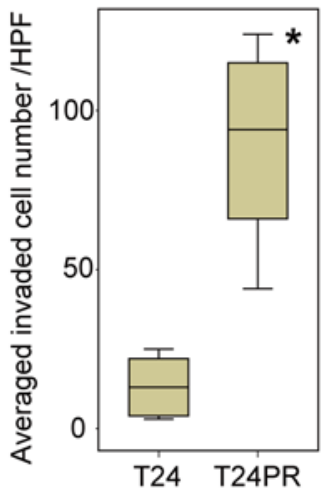

T24 T24PR

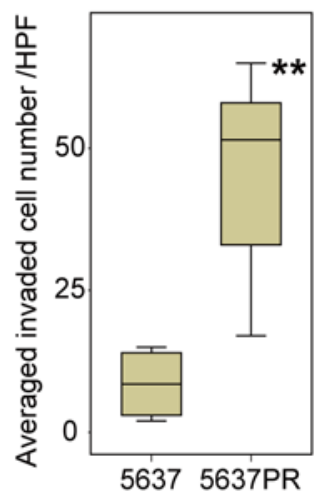

Figure 1. Molecular changes and motile function consistent with epithelial to mesenchymal transition after acquisition of platinum resistance. (A) Western blot analysis of cell lysates for E-cadherin and vimentin and nuclear extracts for SNAIL in T24PR, 5637PR, and their corresponding parent cells, T24 and 5637. (B) Immunofluorescence staining for E-cadherin, vimentin, and SNAIL in T24PR, 5637PR, and their corresponding parent cells, T24 and 5637. (C) Matrigel invasion assay results for T24PR, 5637PR, and their corresponding parent cells, T24 and 5637, at 24 hours $(n=6)$. The invaded cells were stained with Diff-Quik. Box-and-whiskers plots show median (line within box), upper and lower quartile (bounds of box), and minimum and maximum values (bars). HPF, high-power field. ${ }^{*} P<0.05$, compared with T24 cells; ${ }^{* *} P<$ 0.05 , compared with 5637 cells.

We previously reported 2 different UC cell lines with acquired platinum resistance established in our laboratory, T24PR (platinum resistant) and 5637PR (25). In preliminary studies, we observed that these cell lines had an altered phenotype consistent with EMT, and exhibited a marked difference in migratory potential before and after acquired platinum resistance. In the present study, we investigated possible targets associated with EMT in tumors after acquisition of platinum resistance, by analyzing the changes in gene expression patterns of these 2 PR cells.

\section{Results}

Development of acquired platinum resistance involves EMT and the migratory potential. After acquisition of platinum resistance, we observed that T24PR and 5637PR cells exhibited a distinct difference in their lightmicroscopic appearance compared with their corresponding parent cells, T24 and 5637 (Supplemental Figure 1; supplemental material available online with this article; doi:10.1172/jci.insight.83654DS1). The morphological changes observed in both PR cell lines included a spindle-shaped morphology and increased intercellular separation, suggesting that the cells had undergone EMT.

To investigate the specific molecular changes involved with EMT, Western blot (WB) analysis was performed (Figure 1A). Expression of the epithelial adhesion molecule E-cadherin was decreased in T24PR and 5637PR cells compared with their corresponding parent T24 and 5637 cells. In contrast, a marked increase in the expression of the mesenchymal marker vimentin was observed after acquired platinum 
A

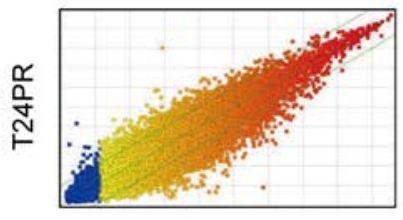

T24

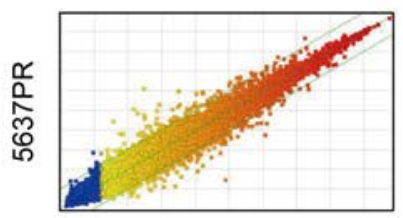

5637
B

Up-regulated

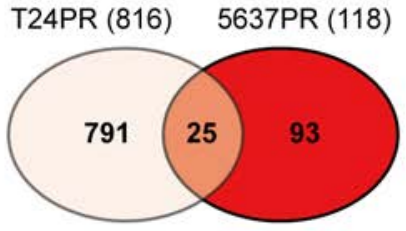

Down-regulated

T24PR (1580) 5637PR (155)

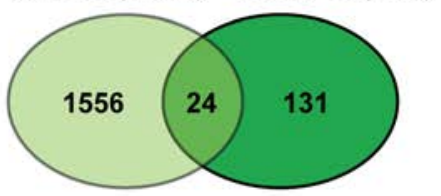

C
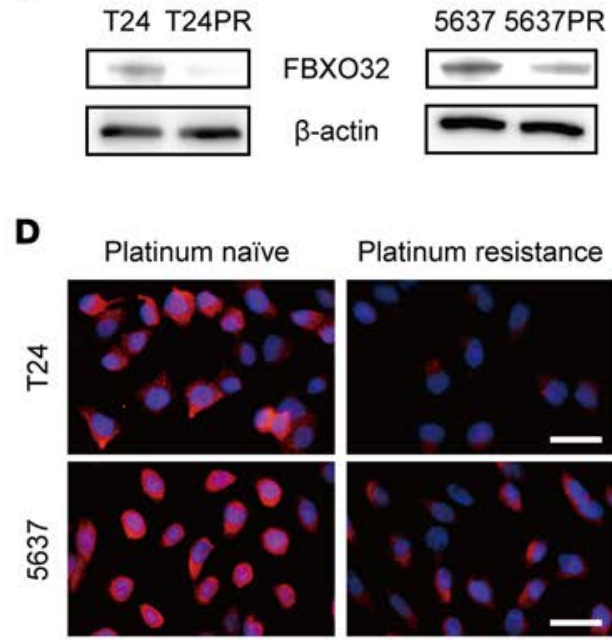

Platinum resistance
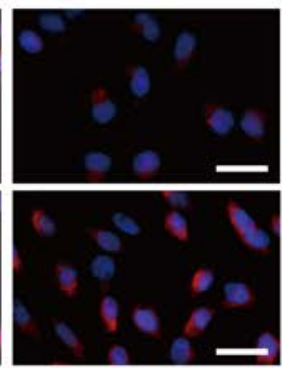

DAPI/FBXO32

\section{E}
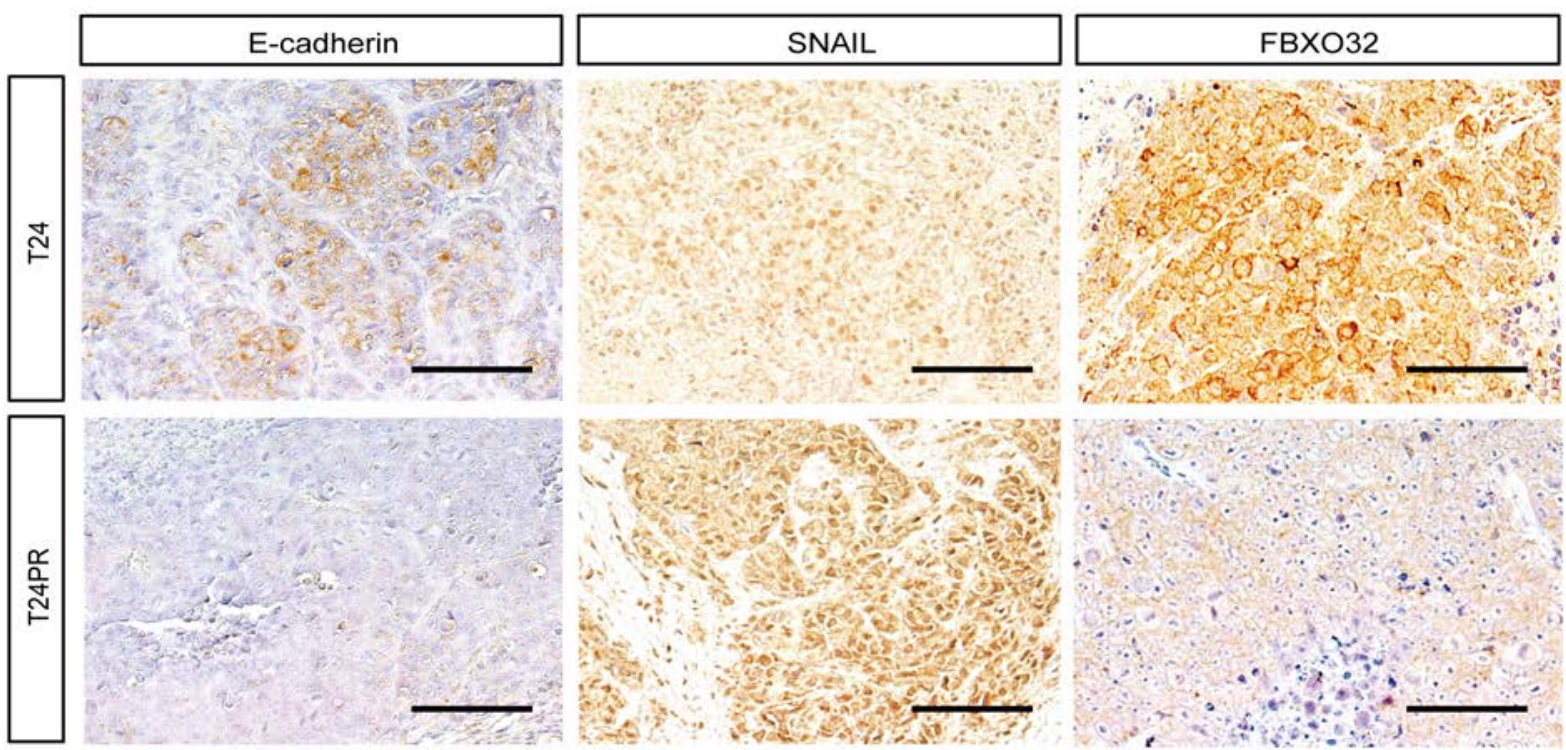

Figure 2. Identification of FBXO32 as a potent negative regulator of the epithelial to mesenchymal transition after acquisition of platinum resistance. (A) Microarray gene expression analysis revealed that the expression of a total of 2,396 genes was changed in T24PR cells (816 upregulated and 1,580 downregulated genes) and 273 genes were changed in 5637PR cells (118 upregulated and 155 downregulated genes) when compared with the corresponding parent cells. The genes with an absolute change $\geq 2$ or $\leq 0.5$ were identified as being differentially expressed. (B) Venn diagram shows common genes between T24PR and 5637PR cells that were either upregulated or downregulated upon acquiring platinum resistance. A total of 49 genes (25 upregulated and 24 downregulated) showed expression changes in both cell lines after they acquired platinum resistance. (C) Western blot analysis of FBX032 in T24PR, 5637PR, and their corresponding parent cells, T24 and 5637. (D) Immunofluorescence staining of FBX032 in T24PR, 5637PR, and their corresponding parent cells, T24 and 5637. (E) Representative images of E-cadherin, SNAIL, and FBXO32 immunohistochemical staining in T24 and T24PR tumors. Scale bars: $100 \mu \mathrm{m}$.

resistance. Furthermore, increased nuclear expression of the EMT-related transcription factor SNAIL was observed in both PR cell lines. These observations were further confirmed by real-time PCR (Supplemental Figure 2) and immunofluorescence (IF) staining (Figure 1B).

Matrigel invasion assay was performed to examine whether acquired platinum resistance induced a motile phenotype (Figure 1C). Both T24PR and 5637PR cells demonstrated a remarkable increase in the number of invading cells compared with T24 and 5637 cells (T24, $13.3 \pm 3.7$ vs. T24PR, $89.5 \pm 13.4, P<$ $0.05 ; 5637,8.5 \pm 2.4$ vs. $5637 \mathrm{PR}, 46.0 \pm 7.3, P<0.05$ ). These results were further confirmed by woundhealing assay (Supplemental Figure 3). 


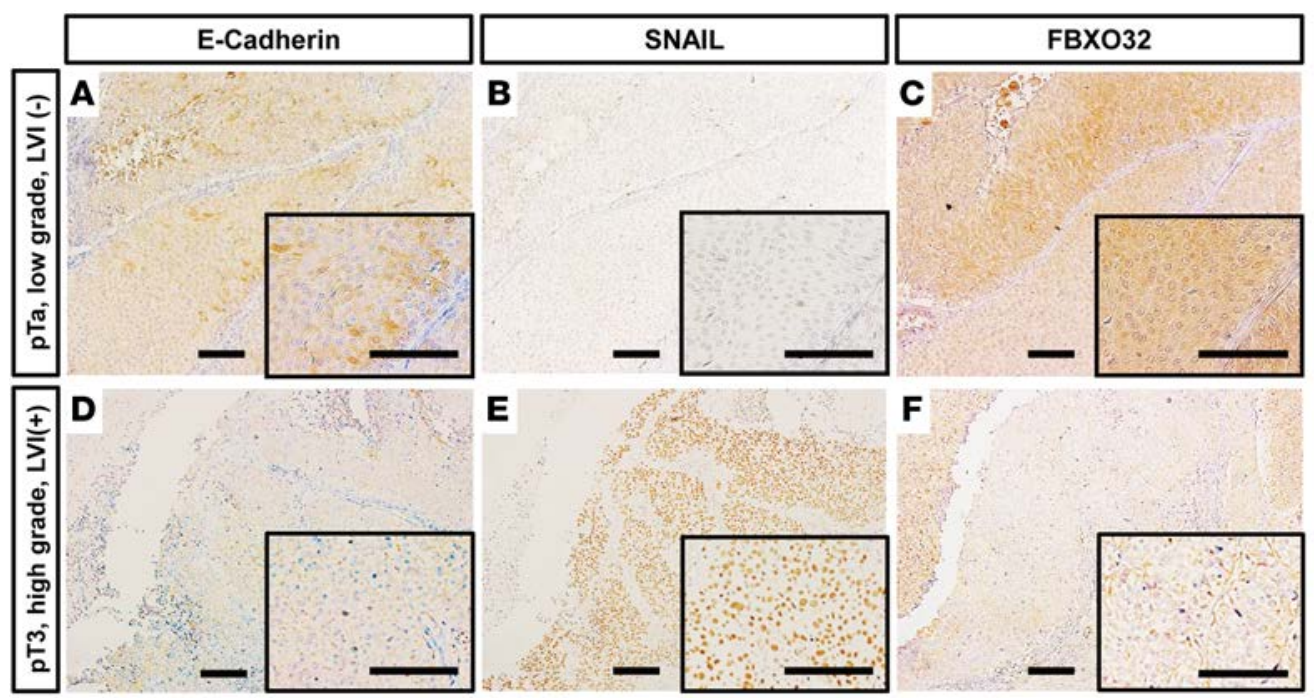

Figure 3. Immunostaining of SNAIL, E-cadherin, and FBXO32 from the same upper urinary tract urothelial carcinoma case. pTa, low-grade, and lymphovascular invasion-negative (LVI-negative) superficial tumor (A, B, and C) and pT3, high-grade, LVI-positive invasive tumor (D, E, and F). Scale bars: $100 \mu \mathrm{m}$.

Newly identified target gene FBXO32 is a negative regulator of EMT after acquisition of platinum resistance. To investigate the possible targets associated with the common phenomenon of EMT in the 2 PR cell lines, we analyzed the changes in global gene expression before and after acquisition of platinum resistance. Comparing the 2 PR cells and their corresponding parent cells, a total of 2,396 genes were changed in T24PR cells (816 upregulated and 1,580 downregulated genes) and 273 were changed in 5637PR cells (118 upregulated and 155 downregulated genes) (Figure 2A).

The Venn diagram in Figure 2B shows the common upregulated/downregulated genes between T24PR and 5637PR cells. Forty-nine common genes (25 upregulated and 24 downregulated genes) were identified with changed expression in both PR cell lines (Supplemental Table 1). Among these 49 genes, we focused on the expression of the muscle-specific F-box protein FBXO32, a member of the SCF family of E3 ubiquitin ligases. FBXO32, also known as atrogin $1 / \mathrm{MAFbx}$, was first identified in models of skeletal muscle atrophy. These studies demonstrated that overexpression of FBXO32 in skeletal myotubes led to atrophy, and mice deficient in FBXO32 were resistant to denervation atrophy of the skeletal muscle (26-31). Also, previous studies have suggested the association between FBXO32 and its substrates and the reduction of EMT-related marker expression during muscle atrophy (32-34). Thus, we hypothesized that the decrease in FBXO32 expression after acquisition of platinum resistance may be associated with the EMT phenotype and migratory potential.

WB and real-time PCR results showed that T24PR and 5637PR cells had significantly lower FBXO32 expression than their corresponding parent cells (Figure 2C and Supplemental Figure 2). Similar results were observed by IF staining (Figure 2D). Furthermore, these results were consistent with in vivo immunohistochemistry assessment (Figure 2E), showing mouse xenograft T24PR tumors with lower FBXO32 expression than that of T24 tumors (T24, $127 \pm 14.4$ vs. T24PR, $68 \pm 5.9, P<0.05$ ). Regarding EMTrelated markers, the expression of SNAIL in T24PR tumors $(150 \pm 11.3)$ was significantly higher than in T24 tumors $(114 \pm 9.5, P<0.05)$, while T24PR tumors showed significantly lower E-cadherin expression $(19 \pm 2.3)$ than T24 tumors $(31 \pm 3.8, P<0.05)$.

Association between EMT, FBXO32 expression, and clinicopathological features in 201 surgically treated patients with upper urinary tract UC. To further examine the association between FBXO32 expression and EMT, we investigated the expression of SNAIL, E-cadherin, and FBXO32 in 201 human upper urinary tract UC specimens (Figure 3, A-F). Immunohistochemical analysis revealed that nuclear SNAIL staining tended to be weak in superficial UCs, while strong nuclear staining of SNAIL was observed in the invasive tumors, as we have previously reported (24). On the other hand, E-cadherin and FBXO32 staining tended to be strong in superficial UCs, while weak staining was observed in the invasive tumors. Table 1 summarizes the 
A

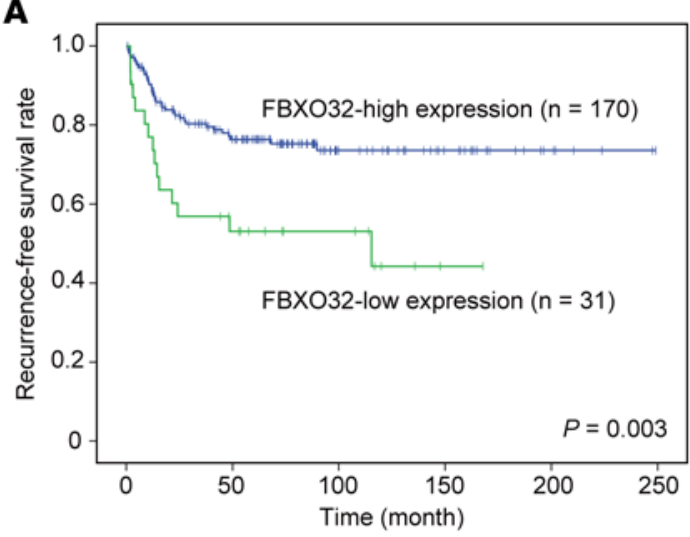

C

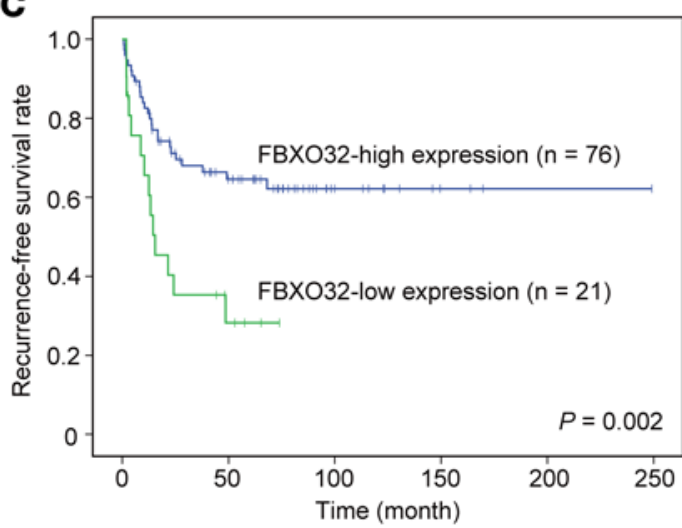

E

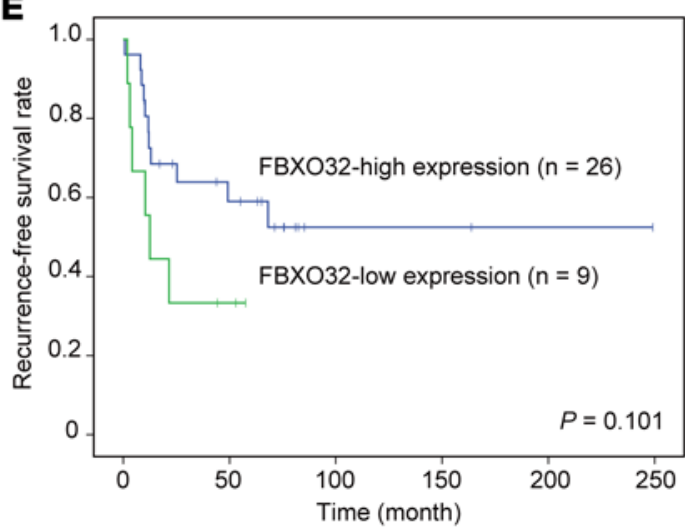

B

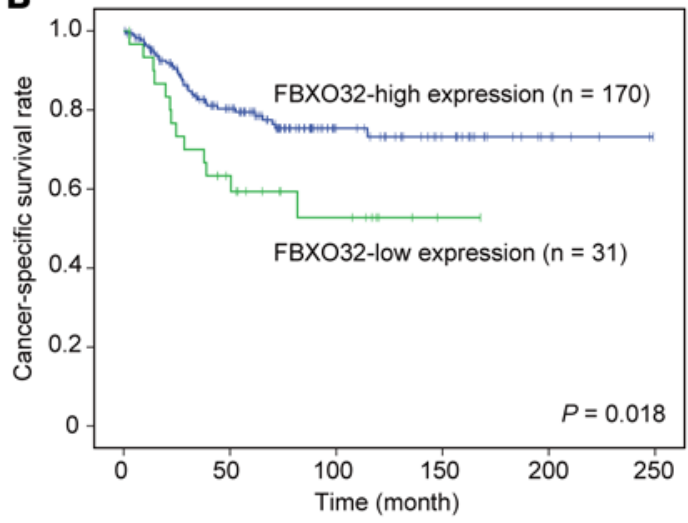

D

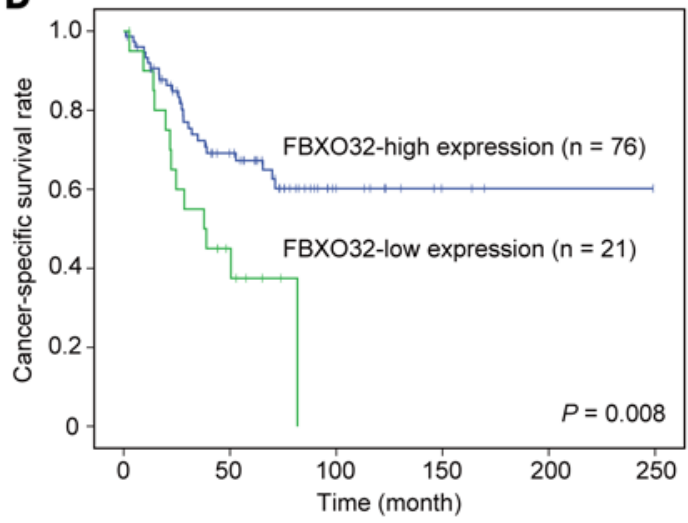

$\mathbf{F}$

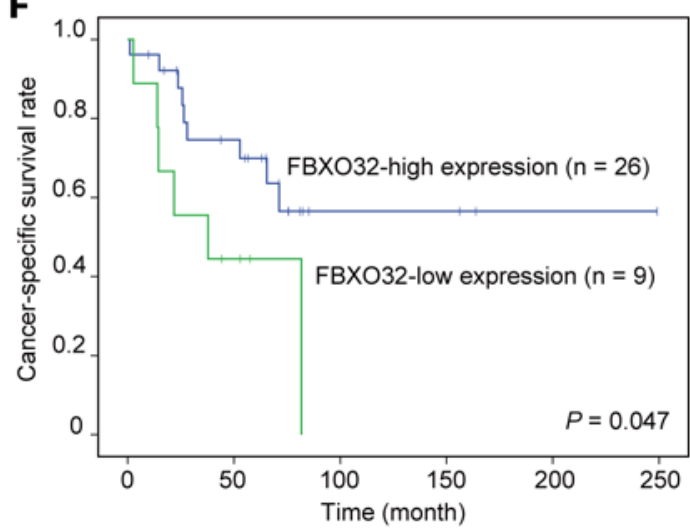

Figure 4. FBX032 expression and survival outcome of surgically treated upper urinary tract urothelial carcinoma patients. (A and B) Kaplan-Meier curves according to FBX032 expression of recurrence-free and cancer-specific survival of 201 patients. (C and D) Kaplan-Meier curves according to FBX032 expression of recurrence-free and cancer-specific survival of 97 patients who had pT3 or greater tumors. (E and F) Kaplan-Meier curves according to FBXO32 expression of recurrence-free and cancer-specific survival of 35 patients who received adjuvant CDDP-based chemotherapy following surgery.

association between SNAIL, E-cadherin, and FBXO32 expression and the clinicopathological features of the 201 subjects. FBXO32 expression was significantly decreased in tumors of a high grade, advanced pT stage, and with positive lymphovascular invasion (LVI).

Prognostic significance of FBXO32 expression. To evaluate the prognostic value of FBXO32 expression, we performed univariate and multivariate analyses to determine the indicators for predicting disease recurrence and cancer-specific mortality in these 201 subjects (Supplemental Table 2). In Kaplan-Meier analysis, decreased FBXO32 expression was shown to be an independent risk factor for predicting disease recurrence $(P=0.015$, hazard ratio $[\mathrm{HR}]=2.11)$ as well as cancer-specific mortality $(P=0.044, \mathrm{HR}$ $=1.93$ ), in addition to other indicators, such as advanced $\mathrm{pT}$ stage and positive LVI. The 5-year recurrence-free and cancer-specific survival rates were $53.1 \%$ and $59.4 \%$ in patients with decreased FBXO32 
Table 1. Baseline characteristics and FBX032 expression of 201 patients treated with radical nephroureterectomy

\begin{tabular}{|c|c|c|c|}
\hline Characteristic & No of patients (\%) & FBX032 score (mean \pm SEM) & $P$ value \\
\hline Age & & & 0.188 \\
\hline$\leq 70$ years & $97(48.3)$ & $92.8 \pm 5.3$ & \\
\hline$>70$ years & $104(51.7)$ & $102 \pm 4.9$ & \\
\hline Gender & & & 0.078 \\
\hline Male & $150(74.6)$ & $101 \pm 4.3$ & \\
\hline Female & $51(25.4)$ & $85.5 \pm 6.1$ & \\
\hline Tumor grade & & & $<0.001$ \\
\hline Low & 54 (26.9) & $119 \pm 7.7$ & \\
\hline High & $147(73.1)$ & $89.5 \pm 3.8$ & \\
\hline Pathological T stage & & & 0.015 \\
\hline $\mathrm{pTa} / 1$ & $70(34.8)$ & $112 \pm 6.1$ & \\
\hline pT2 & 34 (16.9) & $90.9 \pm 6.0$ & \\
\hline рT3 & $90(44.8)$ & $90.2 \pm 5.6$ & \\
\hline pT4 & $7(3.5)$ & $67.1 \pm 18.2$ & \\
\hline Lymphovascular invasion & & & 0.028 \\
\hline Negative & $119(59.2)$ & $104 \pm 4.6$ & \\
\hline Positive & $82(40.8)$ & $88.1 \pm 5.6$ & \\
\hline Lymph node involvement & & & 0.568 \\
\hline $\mathrm{pNx}$ or pNO & $191(95.0)$ & $98.0 \pm 3.7$ & \\
\hline $\mathrm{pN}+$ & $10(5.0)$ & $85.0 \pm 14.8$ & \\
\hline Adjuvant chemotherapy & & & 0.062 \\
\hline No & $163(95.0)$ & $101 \pm 4.0$ & \\
\hline Yes & $38(5.0)$ & $81.7 \pm 7.4$ & \\
\hline E-cadherin expression & & & 0.037 \\
\hline Low & $108(53.7)$ & $91.2 \pm 4.9$ & \\
\hline High & $93(46.3)$ & $104 \pm 5.2$ & \\
\hline SNAIL expression & & & 0.006 \\
\hline Low & $101(50.2)$ & $107 \pm 5.1$ & \\
\hline High & $100(49.8)$ & $87.8 \pm 4.9$ & \\
\hline
\end{tabular}

was significantly decreased in tumors with higher SNAIL expression and lower E-cadherin expression, consistent with previous results in upper urinary tract UCs (Figure 3, A-F, and Table 1). Furthermore, Kaplan-Meier curves of these patients revealed that the 5 -year recurrence-free and cancer-specific survival rates were $44.3 \%$ and $53.7 \%$ in patients with decreased FBXO32 expression, and 73.2\% (Supplemental Figure 4C, $P=0.010$ ) and $80.9 \%$ (Supplemental Figure 4D, $P=0.023)$ in those with higher expression, respectively.

Of the 94 patients with bladder UC, 16 underwent neoadjuvant CDDP-based chemotherapy before cystectomy, and we obtained 16 matched pre- and posttreatment sections (Figure 5, A-F, and Supplemental Table 4). Of the 16 patients, tumors tended to exhibit a higher level of SNAIL expression (Figure 5E, $P$ $=0.012$ ) and a lower level of FBXO32 expression (Figure 5F, $P=0.094$ ) after chemotherapy. Further, 12 tumors (75\%) after chemotherapy exhibited a lower level of FBXO32 expression when comparing with that in the corresponding tumors before chemotherapy. In this group, 9 experienced subsequent disease recurrence and 8 died of disease with a median followup of 34 months after surgery. In Kaplan-Meier analysis, the association with FBXO32 expression and outcome was similar, showing that patients with decreased FBXO32 expression at cystectomy tended to have subsequent disease recurrence (Supplemental Figure 5A, $P=0.058$ ) and cancer-specific mortality (Supplemental Figure 5B, $P=0.092$ ), compared with those with higher expression, respectively.

Effect of FBXO32 knockdown in platinum-naive UC cells. To examine the effects of FBXO32 knockdown, we transfected T24 cells with FBXO32-specific siRNA (si-FBXO32) and evaluated the subsequent changes in EMT. WB analysis showed that si-FBXO32 reduced the level of FBXO32 expression compared with mock-transfected cells (Figure 6A). Furthermore, si-FBXO32 downregulated E-cadherin expression and 

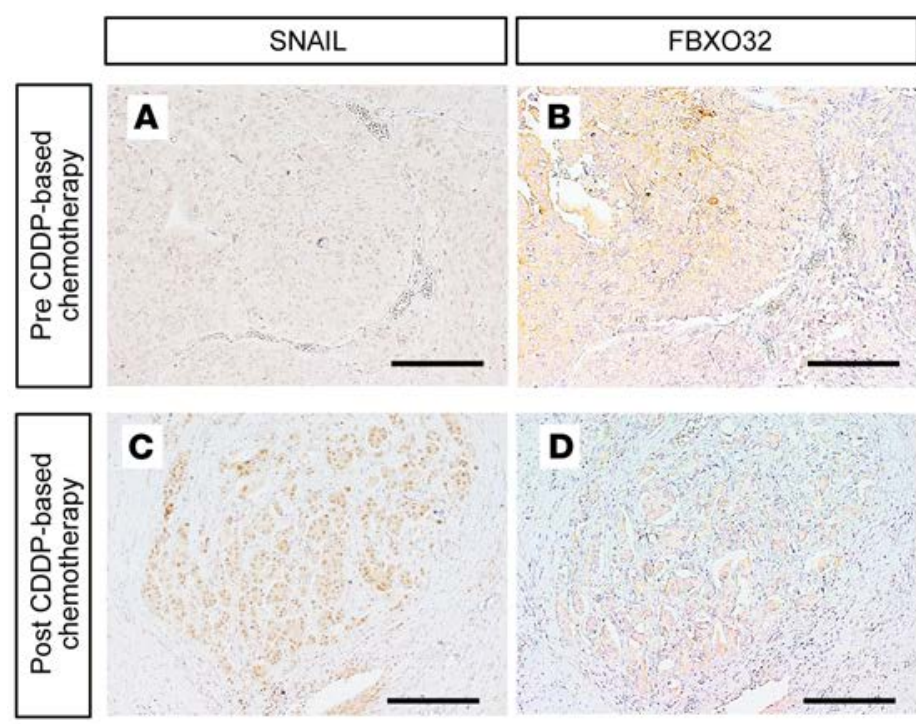
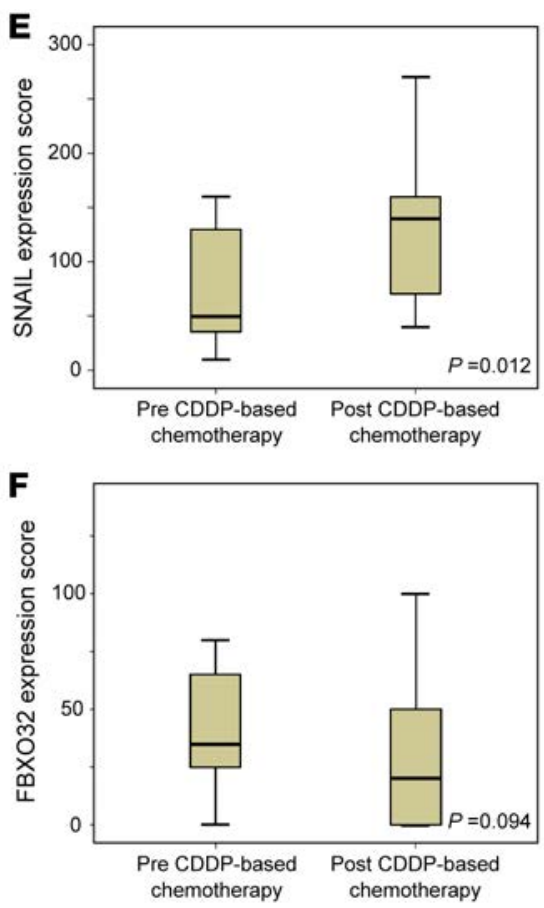

Figure 5. Immunostaining from 16 bladder urothelial carcinoma (UC) patients who underwent neoadjuvant CDDP-based chemotherapy. (A-D) Immunostaining of SNAIL and FBXO32 from the same bladder UC case before and after neoadjuvant CDDP-based chemotherapy. Scale bars: $0.1 \mathrm{~mm}$. (E and F) Box plot of SNAIL and FBXO32 expression from 16 subjects before and after neoadjuvant CDDP-based chemotherapy. The box boundaries represent the upper and lower quartiles, and the horizontal line is the median value. The whiskers represent the minimum and maximum values.

upregulated SNAIL and vimentin expression in T24 cells. Similar results were also observed in real-time PCR (Supplemental Figure 6), and we confirmed these results in 5637 cells (Supplemental Figure 7).

Regarding motile function, Matrigel invasion assay revealed that T24 cells transfected with si-FBXO32 exhibited a significant increase in invasive activity, up to 6-fold greater than mock-transfected cells (Figure 6B). Furthermore, we examined the effect of FBXO32 knockdown on the sensitivity to CDDP (Figure 6C). T24 cells transfected with si-FBXO32 revealed decreased sensitivity to CDDP compared with mock-transfected cells, and similar results were observed in 5637 cells (Supplemental Figure 7). These results indicated a relationship between platinum resistance and decreased FBXO32 expression in cancer cells.

Effect of FBXO32 overexpression in UC cells with acquired platinum resistance. To examine the effects of FBXO32 overexpression after acquisition of platinum resistance, we transfected T24PR cells with FBXO32 and evaluated the subsequent changes in EMT and sensitivity to CDDP. WB analysis showed that transfection of FBXO32 in T24PR cells increased the level of FBXO32 expression compared with control cells. Furthermore, FBXO32 overexpression upregulated the level of E-cadherin in T24PR cells, while SNAIL and vimentin expression was downregulated (Figure 6D). Changes in motile function were assessed by Matrigel invasion assay; T24PR cells overexpressing FBXO32 exhibited a marked decrease in invasion compared with control cells (Figure 6E). Furthermore, we investigated the effect of FBXO32 overexpression on the sensitivity to CDDP, and observed an increased sensitivity to CDDP compared with control cells (Figure 6F).

Effect of knockdown of FBXO32 substrate target MyoD after acquisition of platinum resistance. To further elucidate the details of FBXO32 dysregulation and EMT, we focused on the expression of $\mathrm{MyoD}$, a specific target of FBXO32 polyubiquitination, after acquisition of platinum resistance. WB analysis (Figure 6G) and IF staining (Figure $6 \mathrm{H}$ ) revealed that T24PR cells had significantly elevated MyoD expression compared with that of T24 cells, and similar results were observed in 5637 and 5637PR cells (Supplemental Figure 7). In addition, FBXO32 transfection downregulated the level of MyoD in T24PR cells (Figure 6I).

To examine the effects of MyoD knockdown, we transfected T24PR cells with si-MYOD and evaluated the subsequent changes in EMT (Figure 6J). WB analysis revealed that si-MYOD markedly reduced the level of MyoD expression compared with mock-transfected cells. si-MYOD upregulated the level of 
A

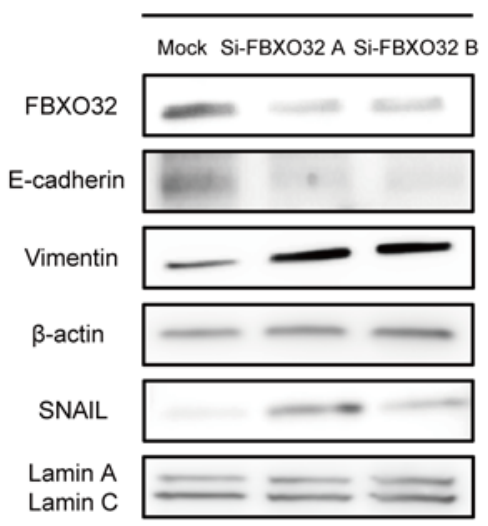

D

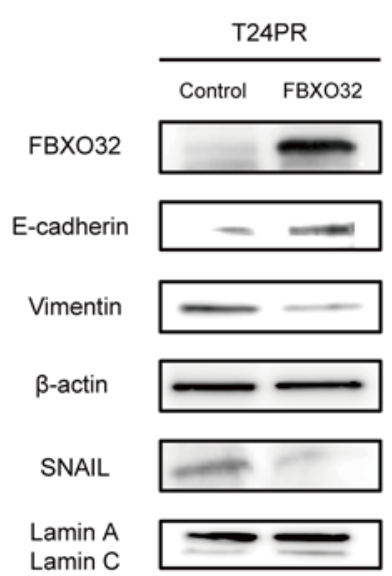

H

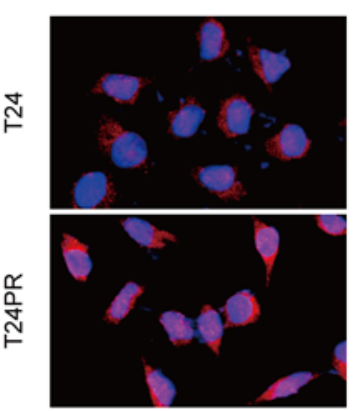

I

DAPI/MyoD

Control $\mathrm{FBXO32}$

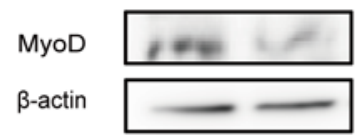

B

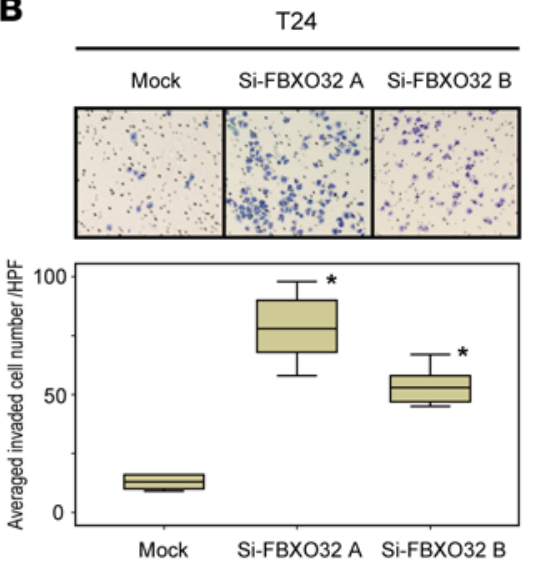

E
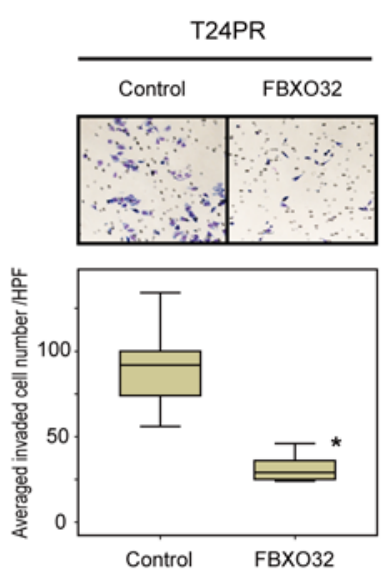

J

T24

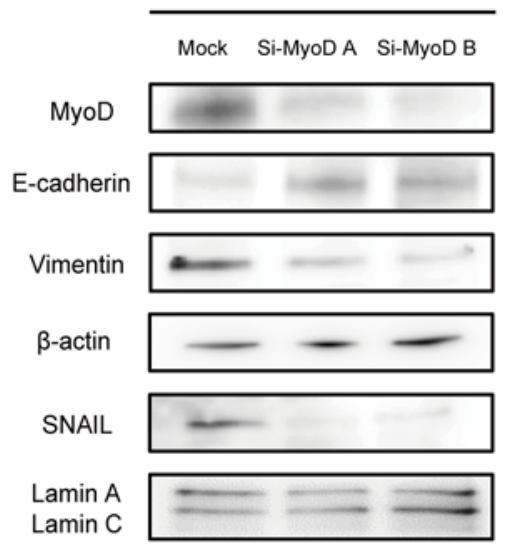

C

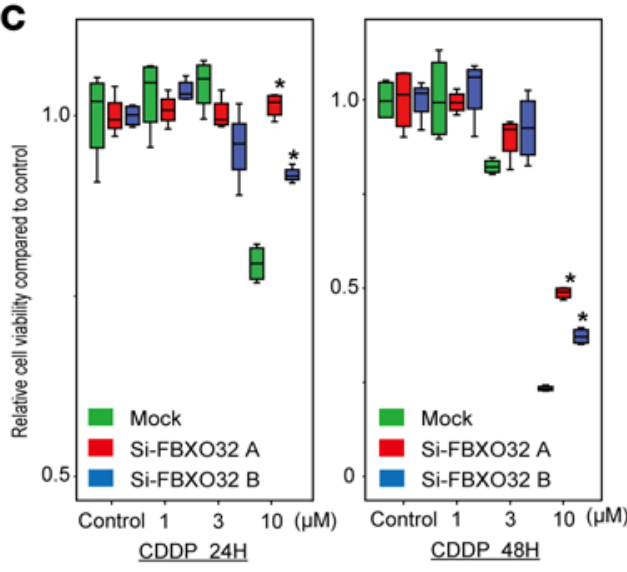

$\mathbf{F}$

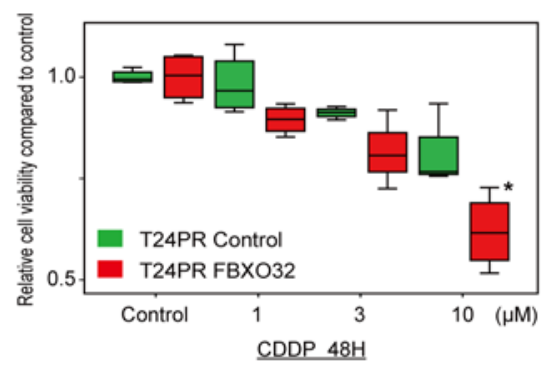

G

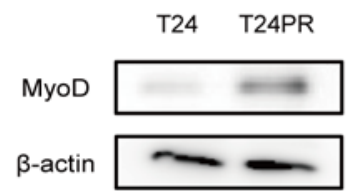

K
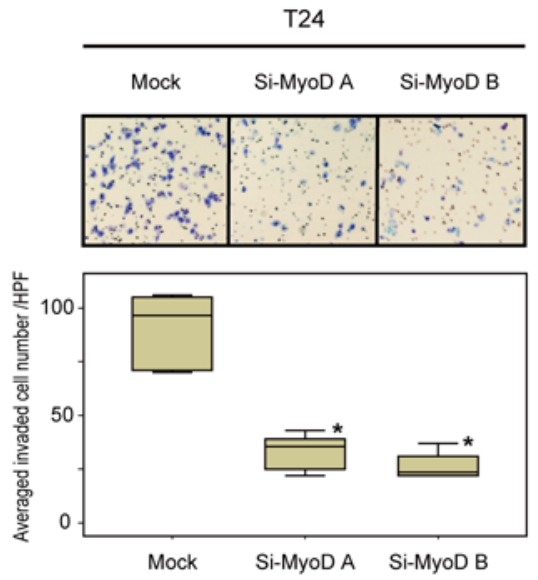

Figure 6. Knockdown and overexpression of FBXO32 and its substrate MyoD. (A) Effect of FBXO32 knockdown on the expression of E-cadherin, vimentin, and SNAIL in T24 cells. (B) Matrigel invasion assay of T24 cells transfected with FBX032-specific siRNA (si-FBXO32) at 24 hours ( $n=6$ ). The invading cells were stained with Diff-Quik. Box-and-whiskers plots show median (line within box), upper and lower quartile (bounds of box), and minimum and maximum values (bars). ${ }^{*} P<0.05,{ }^{* *} P<0.01$, compared with mock-transfected cells. (C) Effect of FBXO32 knockdown on the sensitivity to CDDP in T24 cells. Box-and-whiskers plots show median (line within box), upper and lower quartile (bounds of box), and minimum and maximum values (bars). ${ }^{*} P$ $<0.05$, ${ }^{* *} P<0.01$, compared with mock-transfected cells. (D) Effect of FBX032 overexpression on the expression of E-cadherin, vimentin, and SNAIL in T24PR cells. (E) Matrigel invasion assay of T24PR cells transfected with FBX032 at 24 hours $(n=6)$. The invading cells were stained with Diff-Quik. Box-and-whiskers plots show median (line within box), upper and lower quartile (bounds of box), and minimum and maximum values (bars). ${ }^{*} P<0.05$, compared with control cells. (F) Effect of FBX032 overexpression on the sensitivity to CDDP in T24PR cells. Box-and-whiskers plots show median (line within box), upper and lower quartile (bounds of box), and minimum and maximum values (bars). ${ }^{*} P<0.05$, compared with control cells. (G) Western 
blot analysis of MyoD in T24PR and T24 cells. (H) Immunofluorescence staining for MyoD in T24PR and T24 cells. (I) Effect of FBX032 overexpression on the expression of MyoD in T24PR cells. (J) Effect of MyoD knockdown on the expression of E-cadherin, vimentin, and SNAIL in T24PR cells. (K) Matrigel invasion assay of T24PR cells transfected with si-MYOD at 24 hours $(n=6)$. The invading cells were stained with Diff-Quik. Box-and-whiskers plots show median (line within box), upper and lower quartile (bounds of box), and minimum and maximum values (bars). ${ }^{*} P<0.05$, ${ }^{*} P<0.01$, compared with mock-transfected cells. HPF, high-power field.

E-cadherin in T24PR cells, while vimentin and SNAIL expression in the nucleus was downregulated. These observations were further confirmed by real-time PCR (Supplemental Figure 8). We did not find any significant difference in the level of FBXO32 expression between si-MYOD and mock-transfected groups. Matrigel invasion assay revealed that MYOD-knockdown T24PR cells exhibited a significant decrease in invasive activity compared with mock-transfected cells (Figure 6K).

Array comparative genomic hybridization revealed loss of heterozygosity at 8q24.13 harboring FBXO32 in T24PR cells. To clarify the mechanism underlying the change in FBXO32 expression after acquisition of platinum resistance, we hypothesized that genetic modifications induced during platinum exposure may mediate the subsequent dysregulation of FBXO32 expression in cancer cells. We performed genome-wide array comparative genomic hybridization (CGH) analysis to identify possible chromosome break points, and various DNA gains and losses were newly detected in T24PR cells (Supplemental Figure 9). Moreover, T24PR cells showed loss of heterozygosity $(\mathrm{LOH})$ in regions of chromosomes 8, 9, 15, and 20, all of which were heterozygous in T24 cells (Supplemental Figure 10). We further investigated genetic modifications in the FBXO32 locus 8q24.13. As shown in Figure 7, T24PR cells had a chromosome break at LOH on this locus, suggesting that this may be a possible mechanism underlying the FBXO32 dysregulation observed after acquisition of platinum resistance.

\section{Discussion}

We analyzed 2 different molecular events that occurred after acquisition of platinum resistance. First, the development of acquired platinum resistance involved EMT, resulting in tumor aggressiveness with motile function of cancer cells. Second, from global gene expression profiles using 2 types of PR cells, we identified what we believe to be a novel role for the ubiquitin ligase FBXO32 as a negative regulator of the EMT after acquisition of platinum resistance, providing an additional mechanism distinct from those causing platinum resistance of cancer $(35,36)$.

Over the past decade, many researchers have implicated EMT in chemoresistance (4-11, 24). Kajita et al. reported that E-cadherin transcriptional suppressors could promote resistance to genotoxic stress (4). Using human breast cancer cells, Olmeda et al. described marked increases in the sensitivity to chemotherapies when silencing SNAIL expression (7). Also, several studies have reported EMT acquisition when tumors developed acquired resistance to prior therapy, including platinum treatment (12-19). However, the mechanism underlying the association between acquired platinum resistance and EMT in cancer remains to be understood.

FBXO32 is an E3 ubiquitin ligase upregulated during skeletal muscle atrophy (26-31). Increasing evidence suggests that FBXO32 functions as an apoptosis regulator, and its expression can be inhibited by prosurvival signals $(29,31)$. FBXO32 expression was reported to be transcriptionally silenced in breast, ovarian, and esophageal tumors (37-39). Furthermore, Chou et al. reported the potent ability of FBXO32 to resensitize cancer cells to CDDP, suggesting that the regulation of FBXO32 expression may be crucial for cancer progression (38). Recent studies on the epigenetic regulation of FBXO32 expression in malignancies revealed that efficient apoptosis induction by the chromatin modulator DZNep via trimethylation of $\mathrm{H} 3 \mathrm{~K} 27$ required $\mathrm{FBXO} 32(37,40,41)$.

Additionally, MyoD is a well-known target of FBXO32 polyubiquitination, promoting myogenic activity via the activation of muscle-specific gene expression (33). While these myogenic regulatory factors have been shown to directly or indirectly affect the expression of EMT-related markers $(32,34)$, we focused on the role of FBXO32 as a negative regulator of EMT-like changes in tumors after acquisition of platinum resistance. Our results revealed that FBXO32 dysregulation resulted in elevated SNAIL expression, leading to enhanced migratory potential in UC cells after acquisition of platinum resistance. We then investigated the effect of SNAIL knockdown on the EMT phenotype in T24PR cells with acquired platinum resistance, observing strong phenotypic changes in EMT and tumor invasion (Supplemental Figure 11, A and B). Furthermore, to examine the effect of SNAIL knockdown in FBXO32-depleted cells, we investigated EMT 


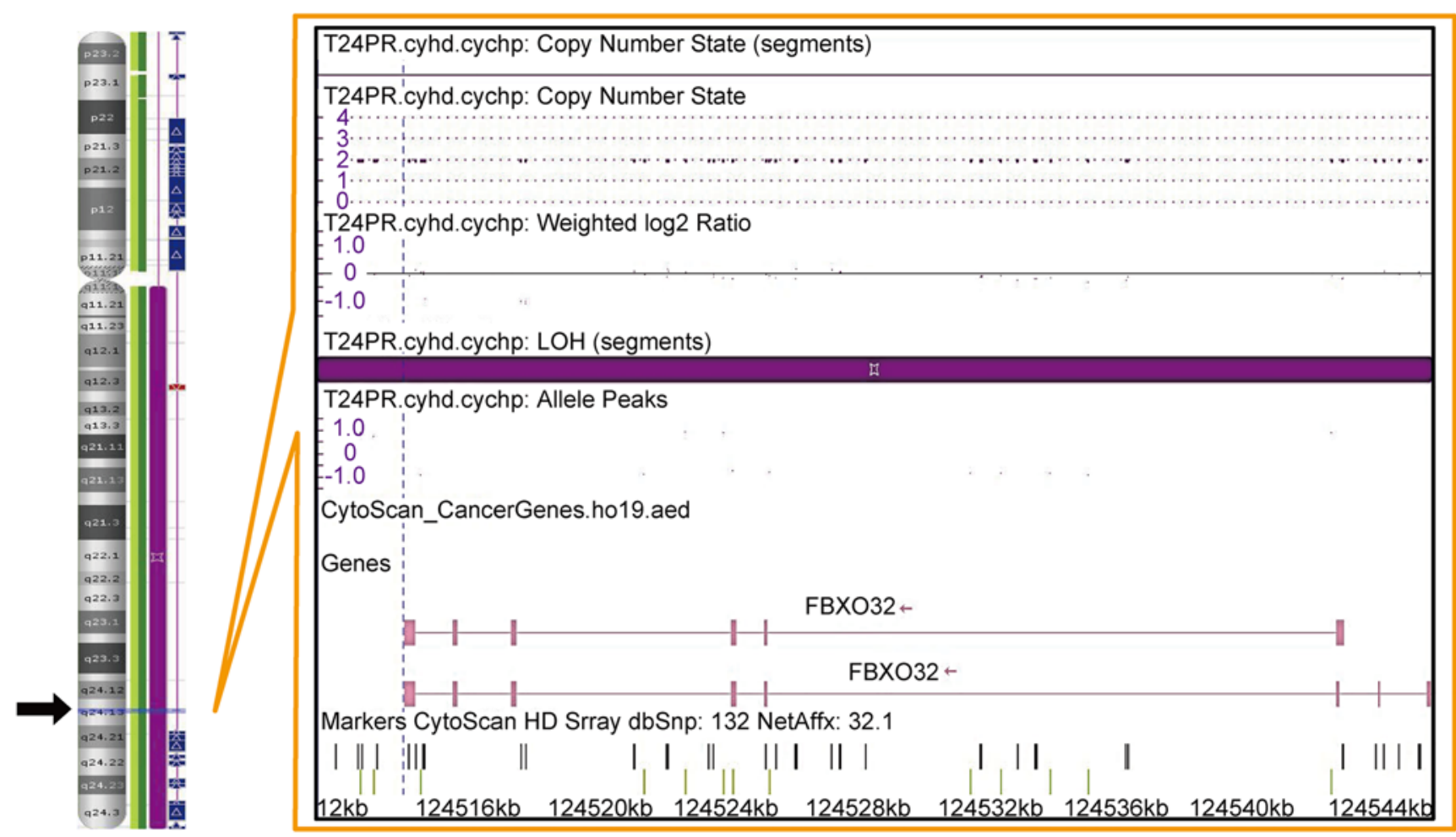

Chr 8

Figure 7. Identification of the possible mechanism of FBX032 dysregulation in T24PR cells with acquired platinum resistance. Array comparative genomic hybridization shows the detected copy number events and loss of heterozygosity in chromosome 8 of T24PR cells. The arrow indicates the FBX032 locus, and color annotation indicates copy number loss (red), copy number gain (blue), and loss of heterozygosity (LOH, purple) in chromosome 8 of T24PR cells.

and tumor invasion in T24 cells cotransfected with si-FBXO32 and si-SNAIL; however, no differences were observed in these cells (Supplemental Figure 11C). These results suggest inverse effects of FBXO32 and SNAIL on EMT acquisition in UC tumors after acquisition of platinum resistance.

To improve our understanding of FBXO32, we studied the impact of enzyme-dead FBXO32 using a mutated FBXO32 (L169Q) $(33,42)$. However, T24PR transfected with mutant FBXO32 did not show any significant changes in EMT-associated molecules or sensitivity to CDDP (Supplemental Figure 12), suggesting the importance of FBXO32 enzymatic activity for the observed EMT-like phenotypic changes and resistance to platinum treatment. In contrast with these findings, we did not find any significant association in our models between FBXO32 expression and other EMT-related transcriptional factors, such as SLUG, TWIST, or ZEB-1 (Supplemental Figure 13). However, while computational analyses using SABiosciences' Text Mining Application and the UCSC Genome Browser on the SLUG, TWIST, and ZEB-1 promoter regions identified the presence of MyoD DNA-binding sites, further investigation is needed to clarify the precise interaction between the FBXO32 and/or MyoD axis and EMT in malignancies.

To determine the mechanism underlying the change in FBXO32 expression, we investigated the effect of CDDP on DNA integrity (43). By array CGH, we newly identified a chromosome break of LOH at the FBXO32 locus in T24PR cells. While CDDP remains one of the most effective agents for treatment of some cancer types owing to its potent genotoxic activity, such a paradoxical effect is particularly interesting in terms of how cancer cells can gain growth and survival benefits during platinum treatment.

Although our findings showed a relationship between FBXO32 dysregulation and EMT in PR UCs, we further considered whether such a phenomenon was limited to only platinum treatment. In metastatic or recurrent patients with UC, other chemotherapeutic agents, such as paclitaxel, have been clinically demonstrated to be an alternative treatment option (1). Thus, we generated the T24TR (taxane-resistant) cell line, a subline of T24 with acquired taxane resistance, and evaluated the subsequent EMT-like changes (Supplemental Figure 14). However, we did not find any significant differences between T24 and T24TR cells in light-microscopic appearance. Furthermore, we did not find any significant differences in the expression of 
E-cadherin, vimentin, SNAIL, or FBXO32 between these 2 cell lines. The results were further confirmed by Matrigel invasion assay, which showed no significant difference in the number of invading cancer cells between the T24 and T24TR cells. Therefore, in our models, the acquisition of the EMT phenotype may occur only after platinum treatment.

However, one limitation of the present study was that we did not evaluate an association between FBXO32 expression and other anticancer agents, such as gemcitabine and molecular targeted agents, which have all been reported to involve EMT-like changes in acquired resistance models of other cancers (13-16, $44,45)$. Other limitations include the limited number of clinical samples, owing to the retrospective nature of the study, as well as the heterogeneity of the patients, owing to factors such as administration of perioperative chemotherapy in some cases. We did not repeat a microarray analysis in our in vitro study. Furthermore, at present, it remains unclear whether EMT itself plays a central role in the development of acquired chemoresistance in patients with UC. Thus, further investigations to more accurately determine the role of FBXO32 in EMT and associated chemoresistance are warranted.

In summary, development of acquired platinum resistance involved an EMT with elevated migratory potential in UC. FBXO32 expression level was associated with the regulation of EMT, showing that FBXO32 dysregulation induced EMT-like changes in the 2 PR cells. Further, knockdown of the FBXO32 target substrate MyoD suppresses EMT phenotypically. Our findings suggest the importance of the association between EMT and ubiquitin-proteasome regulation in UC tumors after acquisition of platinum resistance.

\section{Methods}

Cell lines and culture conditions. Two UC cell lines (T24 and 5637) were obtained from the American Type Culture Collection, and T24PR and 5637PR cells were established in our laboratory as sublines of T24 and 5637 with acquired platinum resistance (25). Briefly, T24 and 5637 cells were grown and passaged upon reaching confluence in medium containing CDDP over a 6-month period to develop platinum resistance, with a gradual increase in CDDP concentration to $3 \mu \mathrm{M}$. T24 and 5637 cells were also continuously cultured and passaged upon reaching confluence during the study period. T24TR cells were newly established for this study as a subline of T24 with acquired taxane resistance by passaging cells in medium containing paclitaxel over a 6-month period to develop taxane resistance. The concentration of paclitaxel was gradually increased to $60 \mathrm{nM}$.

All cells were routinely maintained in RPMI 1640 (Invitrogen) supplemented with 10\% FBS (Dainippon Pharmaceutical) at $37^{\circ} \mathrm{C}$ in a humidified $5 \% \mathrm{CO}_{2}$ atmosphere, and further examinations were performed after 3 months without CDDP/paclitaxel exposure.

Drugs and antibodies. CDDP and paclitaxel were generously supplied by Nippon Kayaku Co. Monoclonal and polyclonal antibodies for E-cadherin (catalog ab1416), vimentin (catalog ab8978), SNAIL (catalog ab180714, ab85931), SLUG (catalog ab27568), FBXO32 (catalog ab74023), and MyoD (catalog ab16148, ab64159) were obtained from Abcam. Monoclonal antibody for E-cadherin (catalog 3195) was purchased from Cell Signaling Technology. Monoclonal antibody for $\beta$-actin (catalog A5316) was purchased from Sigma-Aldrich. Monoclonal and polyclonal antibodies against antibody for lamin A and C (catalog sc-7292), SNAIL (catalog sc-28199), ZEB-1 (catalog sc-10572), and FBXO32 (catalog sc-166806) were purchased from Santa Cruz Biotechnology. Monoclonal antibody for TWIST (catalog BMR00383) was purchased from Bio Matrix Research.

Construction of FBXO32 expression vectors and transfection. Human FBXO32 cDNA $(1,086 \mathrm{bp})$ was synthesized with reference to the human FBXO32 sequence registered in the GeneBank database (http://www.ncbi.nlm.gov/genbank/), accession number NM_58229, and then subcloned into the BamHI-XbaI sites of the pBApo-CMV Neo vector (Takara Bio). The FBXO32 expression vector or control empty vector was transfected into UC cells using TranaIT-2020 Transfection Reagent (Mirus Bio) according to the manufacturer's instructions. After 24 to 72 hours, the cells were used for further experimentation. We also constructed an expression vector with a leucine to glutamine mutation at position 169 of FBXO32 (L169Q) (33).

Murine xenograft bladder cancer model. Six-week-old athymic nude BALB/C mice with an average body weight of $20 \mathrm{~g}$ were obtained from Sankyo Lab Service. T24 and T24PR cells $\left(2 \times 10^{6}\right.$ cells), suspended in $100 \mu 1$ of Matrigel (Becton Dickinson Labware), were implanted subcutaneously into the flank of each mouse. The animals were carefully monitored, and on the 28th day, the animals were sacrificed and the subcutaneous tumors were harvested. 
Cell extracts and WB analysis. Whole-cell extracts were obtained using RIPA buffer (Cell Signaling Technology Japan) containing protease inhibitors, according to the manufacturer's protocol. Total protein lysate $(50 \mu \mathrm{g})$ in sample buffer containing 2-mercaptoethanol was resolved by $4 \%-15 \%$ SDS-PAGE, and transferred to a nitrocellulose or polyvinylidene difluoride membrane, as appropriate (Bio-Rad Laboratories), and then incubated with 5\% skim milk overnight. Primary antibodies were then applied at room temperature for 1 hour. The membranes were then incubated with the appropriate secondary antibodies. Signals were detected by an enhanced chemiluminescence reagent (ECL plus Western Blotting Detection System; Amersham Pharmacia Biotech), and analyzed. Signal intensity was quantified using an LAS 3000 system (Fuji Film). $\beta$-Actin and lamin A and C were used as internal protein controls.

Morphological and IF analyses. To evaluate differences in morphologies, cells were grown to $70 \%$ confluence and assessed at $\times 20$ magnification under a light microscope with digital photographic capability. To evaluate IF staining, $4 \times 10^{4}$ cells were seeded on 14-mm coverslips in 8-well plates. After 48 hours of incubation, the cells were washed with PBS, fixed in 4\% paraformaldehyde, and permeabilized in cold PBS with $0.2 \%$ Triton X-100 at room temperature. After blocking with $3 \%$ bovine serum albumin in PBS, the slides were then incubated with primary antibody for 1 hour at room temperature followed by Alexa 555conjugated secondary antibody. Coverslips were mounted on glass slides in Vectashield mounting medium containing DAPI, and visualized by fluorescence microscopy.

Real-time PCR. Total RNA was isolated using RNAzol (Takara Bio) according to the manufacturer's instructions. RNA quantity and quality were evaluated by spectrophotometry (Nanodrop ND-2000 spectrophotometer, Thermo Scientific). Reverse transcription of RNA to cDNA was performed using the High Capacity cDNA Archive kit (Applied Biosystems). Quantitative gene expression was performed for SNAIL (Hs00195591_m1), E-cadherin (Hs01023894_m1), Vimentin (Hs00185584_4m1), FBXO32 (Hs01041408_ m1), MYOD (Hs00159528_m1), and GAPDH(Hs99999905_m1) with gene-specific probes (Applied Biosystems) using TaqMan Universal PCR Master Mix and the 7500 Fast Real-time PCR system (Applied Biosystems). Cycling conditions were described previously (24), and data were quantified using the comparative $\mathrm{C}_{\mathrm{t}}$ method for relative gene expression, using GAPDH as an endogenous control.

In vitro cell invasion assay. Invasion of tumor cells was assessed by counting the number of cells that migrated through transwell inserts with a polyethylene terephthalate membrane (8- $\mu \mathrm{m}$ pore size) coated with a uniform layer of BD Matrigel Basement Membrane Matrix (BD Biosciences), according to the manufacturer's protocol. Briefly, cells $\left(5 \times 10^{4}\right)$ suspended in $0.5 \mathrm{ml}$ RPMI 1640 with $1 \%$ FBS were seeded in the upper chamber, and the lower chamber was loaded with $0.75 \mathrm{ml}$ of media containing $10 \% \mathrm{FBS}$. After 24 hours, cells that had invaded through to the reverse side of the inserts were rinsed and counterstained using the Diff-Quik stain kit (Sysmex Co.). The invaded cells were counted in 6 randomly chosen visual fields at $\times 10$ magnification. In the wound-healing assay, the migratory potential of cells was assessed by measurement of the width of the scratches at each time point. A scratch was made using a pipet tip after reaching confluence, and visualized with a light microscope with digital photographic capability at 0 and 24 hours.

Microarray gene expression analysis. Total RNA was isolated from cell lines using TRIzol (Invitrogen) and purified by the RNeasy Mini kit (Qiagen). Gene expression profiles were determined using the Affymetrix GeneChip Human Gene 1.0 ST array according to the manufacturer's instructions. After generating the single-stranded cDNA, fragmentation and sense-strand cDNA labeling was performed with the Affymetrix GeneChip WT Terminal Labeling Kit (Affymetrix) according to the manufacturer's protocol. After hybridization, the GeneChip Fluidics Station 450 (Affymetrix) was used for washing the arrays, and scanning was performed with the GeneChip Scanner $30007 \mathrm{G}$ (Affymetrix). The raw intensity data from scanned images of the microarrays were preprocessed using Affymetrix Expression Console software. Expression intensities were stored as cell intensity (CEL) files, and the CEL files were normalized with the robust multichip average method. These datasets were filtered, and genes with an absolute fold change greater than or equal to 2 or less than or equal to 0.5 were identified as being differentially expressed. This microarray data set has been approved by the Gene Expression Omnibus (GEO) (http://www.ncbi.nlm.gov/geo/); its accession number is GSE58624.

Patient selection and tissue samples. Surgical specimens from patients who had been surgically treated for UC of the upper urinary tract $(n=201)$ and bladder $(n=94)$ at Keio University Hospital were used for this study. Patients with prior radiation therapy before surgery and with distant metastasis at the time of surgery were excluded. In this population, 16 patients with bladder UC were treated with neoadjuvant chemotherapy. The main procedure in upper urinary tract UC patients was radical nephroureterectomy with 
removal of the bladder cuff, and partial ureterectomy was performed in 3 patients. Dissection of regional lymph nodes was performed at the attending physician's discretion; however, lymph node dissection was generally performed if enlarged nodes were suspected by preoperative evaluation or intraoperative inspection. The median followup period for all patients with upper urinary tract UC was 57 months (interquartile range [IQR], 26 to 96) and 35 months (IQR, 10 to 63) for patients with bladder UC.

Patients were generally followed up every 3 to 4 months for 2 years following surgery, every 6 months for the next 3 years, and then every 6 to 12 months thereafter. Followup consisted of taking history, physical examination, routine blood work, and/or chest radiography. Radiographic evaluation consisting of computerized tomography (CT), magnetic resonance imaging, and/or excretory urograms were assessed every 6 months for the first 5 years, and annually thereafter. Elective bone scans and chest CT were performed when clinically indicated. Disease recurrence was defined as any documented recurrence by radiographic or pathological proven failure, including local and distant sites. Cause of death was determined by the attending physician, chart review corroborated by death certificates, or by death certificates alone.

Surgical specimens were fixed in $10 \%$ formalin and embedded in paraffin, and all slides were reassessed by a genitourinary pathologist for the present study. All specimens were histologically confirmed to be UC. Tumors were staged according to the 2002 TMN classification and graded according to the 2004 WHO classification. LVI was defined as the presence of tumor cells within the endothelium-lined space without underlying muscular walls.

Immunostaining for E-cadherin, SNAIL, and FBXO32. Formalin-fixed paraffin-embedded sections $(4 \mu \mathrm{m})$ were deparaffinized, rehydrated, and washed in distilled water. After antigen retrieval, endogenous peroxidase was quenched and sections were blocked with skim milk. Primary antibodies against E-cadherin (1:100 dilution; Abcam), SNAIL (1:400 to 1:800 dilution; Abcam), and FBXO32 (1:100 dilution; Abcam) were then applied at room temperature for 1 hour. After washing, the sections were incubated with secondary antibodies against mouse and rabbit IgG conjugated to a peroxidase-labeled dextran polymer for 1 hour. Color was developed with 3,3'-diaminobenzidine in $50 \mathrm{mM}$ Tris- $\mathrm{HCl}$ ( $\mathrm{pH} 5.5$ ) containing $0.005 \%$ hydrogen peroxide. The sections were counterstained with hematoxylin.

Evaluation of SNAIL and E-cadherin staining was previously described (24). Briefly, expression of SNAIL was assessed using the following formula: mean percentage of cancer cells with positive nucleus staining $\times$ highest intensity from 0 to 3 ( 0 , no staining; 1 , slight staining; 2 , medium staining; 3 , strong staining), while E-cadherin expression in the tumor cells was scored as the average of detectable immunoreactions. SNAIL and E-cadherin expression cutoff points were set to the median score of the study population. To evaluate FBXO32 expression, the intensity of FBXO32 staining in tumor lesions was stratified from 0 to 3 ( 0 , no staining; 1 , slight staining; 2, medium staining; 3 , strong staining), and the immunopositivity of stained cancer cells was assessed in at least 10 representative areas at $\times 10$ magnification. Finally, the highest histoscore (FBXO32 score) was calculated by applying the following formula: mean percentage $\times$ highest intensity (range $0-300$ ). In this study, the cutoff value of a FBXO32 score less than or equal to 40 was defined as decreased. Two authors blinded to the patient data independently evaluated the immunoreactivity.

siRNA. FBXO32 and MyoD expression was transiently downregulated using the following predesigned duplex siRNA. The sense sequences of the siRNAs were as follows: si-FBXO32A, 5'-CUUGUCCGAUGUUACCCAATT-3'; si-FBXO32B, 5'-GCAGAUCCGCAAACGAUUATT-3'; $\quad$ si- $M Y O D A$, 5'-CAAAGUAAAUGAGGCCUUUTT-3'; si-MYODB, 5'-GCACGUCGAGCAAUCCAAATT-3'. Cells were transiently transfected with $20 \mathrm{nmol}$ of the respective siRNAs using Lipofectamine 2000 (Life Technologies). After 12 hours, siRNA was removed by replacing the culture medium with fresh RPMI 1640 containing 10\% FBS, and the cells were further incubated for 36 hours. A mock-transfected control was prepared using the transfection reagent only.

Cell viability assay. All cell lines were seeded at a density of $3 \times 10^{3}$ per well in 96-well culture plates. Following a 24-hour incubation in RPMI 1640 medium with $10 \%$ FBS, the cells were incubated for the appropriate time with various concentrations of CDDP or paclitaxel to investigate the sensitivity of the cell lines to each agent. At the end of the incubation period, cell viability was determined using the Premix WST-1 Cell Proliferation Assay System (Takara Bio). The absorbance value of each well was determined in a microplate spectrophotometer (Bio-Rad Laboratories).

Array CGH analysis. To detect genome-wide copy number abnormalities and LOH, total DNA was iso- 
lated from cell lines using TRIzol and purified using the DNeasy Mini Kit. The samples were then analyzed by Affymetrix CytoScan HD array according to the manufacturer's protocol. After fragmentation and DNA labeling, the GeneChip Fluidics Station 450 was used to wash the arrays. Scanning was performed with the GeneChip Scanner 3000 7G. The raw intensity data from scanned images of the microarrays were preprocessed using GeneChip Command Console software (Affymetrix), and the expression intensities were stored as CEL files. The copy number and genotyping analyses were performed using Affymetrix Chromosome Analysis Suite software according to the manufacturer's protocol.

Statistics. The values are given as the mean \pm SEM, median, and interquartile range for continuous variables, and frequency with percentage for categorical variables. Variables between groups were compared using the Mann-Whitney $U$ test and Kruskal-Wallis test, as appropriate. Survival curves were estimated using the Kaplan-Meier method and compared using the log-rank test. Univariate and multivariate Cox regression models were used to evaluate time to disease recurrence and cancer-specific mortality. All parameters were included in the multivariate analyses. Differences among groups were regarded as significant when $P<0.05$. All analyses were performed using the SPSS version 22.0 statistical software package.

Study approval. All animal studies were approved by the Laboratory Animal Center, Keio University School of Medicine. All human studies were reviewed and approved by the Institutional Review Board, Keio University School of Medicine.

\section{Author contributions}

NT and TK developed the project, performed experiments, analyzed data, and wrote the manuscript with the supervision of MO. YM, NN, YO, and YM performed experiments and analyzed data. SM, RM, EK, and $\mathrm{AM}$ assisted in concept development. HS, YO, PU, and MS provided their expert advice in the development of the project and assisted in editing the manuscript.

\section{Acknowledgments}

The authors would like to sincerely thank Ms. Yukiko Nakajima for her assistance with the experiments. This study was supported in part by Grants-in-Aid for Scientific Research (grants 24791671 and 26462429 to N. Tanaka, 24890230 and 26861299 to T. Kosaka, and 26670163 to Y.A. Minamishima) from the Ministry of Education, Culture, Sports, Science, and Technology of Japan. The study was supported in part by a research grant to N. Tanaka from the Takeda Science Foundation, Japan. Also, the study was supported in part by a research grant to N. Tanaka from the Uehara Memorial Foundation, Japan. M.S. is the leader of CREST, JST, Japan for FY2014.

Address correspondence to: Mototsugu Oya, or Takeo Kosaka, Department of Urology, Keio University School of Medicine, 35 Shinanomachi, Shinjuku-ku, Tokyo, 160-8582, Japan. Phone: 81.3.5363.3825; E-mail: moto-oya@z3.keio.jp (M. Oya) or takemduro@gmail.com (T. Kosaka).

1. Witjes JA, et al. EAU guidelines on muscle-invasive and metastatic bladder cancer: summary of the 2013 guidelines. Eur Urol. 2014;65(4):778-792.

2. Rouprêt M, et al. European guidelines on upper tract urothelial carcinomas: 2013 update. Eur Urol. 2013;63(6):1059-1071.

3. von der Maase $\mathrm{H}$, et al. Long-term survival results of a randomized trial comparing gemcitabine plus cisplatin, with methotrexate, vinblastine, doxorubicin, plus cisplatin in patients with bladder cancer. J Clin Oncol. 2005;23(21):4602-4608

4. Kajita M, McClinic KN, Wade PA. Aberrant expression of the transcription factors snail and slug alters the response to genotoxic stress. Mol Cell Biol. 2004;24(17):7559-7566

5. Vega S, Morales AV, Ocaña OH, Valdés F, Fabregat I, Nieto MA. Snail blocks the cell cycle and confers resistance to cell death. Genes Dev. 2004;18(10):1131-1143.

6. Baumgart E, et al. Identification and prognostic significance of an epithelial-mesenchymal transition expression profile in human bladder tumors. Clin Cancer Res. 2007;13(6):1685-1694.

7. Olmeda D, Moreno-Bueno G, Flores JM, Fabra A, Portillo F, Cano A. SNAI1 is required for tumor growth and lymph node metastasis of human breast carcinoma MDA-MB-231 cells. Cancer Res. 2007;67(24):11721-11731.

8. Black PC, et al. Sensitivity to epidermal growth factor receptor inhibitor requires E-cadherin expression in urothelial carcinoma cells. Clin Cancer Res. 2008;14(5):1478-1486.

9. Arumugam T, et al. Epithelial to mesenchymal transition contributes to drug resistance in pancreatic cancer. Cancer Res. 2009;69(14):5820-5828.

10. McConkey DJ, et al. Role of epithelial-to-mesenchymal transition (EMT) in drug sensitivity and metastasis in bladder cancer. Cancer Metastasis Rev. 2009;28(3-4):335-344. 
11. Latifi A, et al. Cisplatin treatment of primary and metastatic epithelial ovarian carcinomas generates residual cells with mesenchymal stem cell-like profile. J Cell Biochem. 2011;112(10):2850-2864.

12. Yang AD, et al. Chronic oxaliplatin resistance induces epithelial-to-mesenchymal transition in colorectal cancer cell lines. Clin Cancer Res. 2006;12(14 Pt 1):4147-4153.

13. Shah AN, Summy JM, Zhang J, Park SI, Parikh NU, Gallick GE. Development and characterization of gemcitabine-resistant pancreatic tumor cells. Ann Surg Oncol. 2007;14(12):3629-3637.

14. Wang Z, et al. Acquisition of epithelial-mesenchymal transition phenotype of gemcitabine-resistant pancreatic cancer cells is linked with activation of the notch signaling pathway. Cancer Res. 2009;69(6):2400-2407.

15. Güngör C, et al. Notch signaling activated by replication stress-induced expression of midkine drives epithelial-mesenchymal transition and chemoresistance in pancreatic cancer. Cancer Res. 2011;71(14):5009-5019.

16. Rosanò L, et al. Acquisition of chemoresistance and EMT phenotype is linked with activation of the endothelin A receptor pathway in ovarian carcinoma cells. Clin Cancer Res. 2011;17(8):2350-2360.

17. Haslehurst AM, et al. EMT transcription factors snail and slug directly contribute to cisplatin resistance in ovarian cancer. $B M C$ Cancer. 2012;12:91.

18. Barr MP, et al. Generation and characterisation of cisplatin-resistant non-small cell lung cancer cell lines displaying a stem-like signature. PLoS One. 2013;8(1):e54193.

19. Kurokawa M, Ise N, Omi K, Goishi K, Higashiyama S. Cisplatin influences acquisition of resistance to molecular-targeted agents through epithelial-mesenchymal transition-like changes. Cancer Sci. 2013;104(7):904-911.

20. Thiery JP. Epithelial-mesenchymal transitions in tumour progression. Nat Rev Cancer. 2002;2(6):442-454.

21. Barrallo-Gimeno A, Nieto MA. The Snail genes as inducers of cell movement and survival: implications in development and cancer. Development. 2005;132(14):3151-3161.

22. Peinado H, Olmeda D, Cano A. Snail, Zeb and bHLH factors in tumour progression: an alliance against the epithelial phenotype? Nat Rev Cancer. 2007;7(6):415-428.

23. De Craene B, Berx G. Regulatory networks defining EMT during cancer initiation and progression. Nat Rev Cancer. 2013;13(2):97-110.

24. Kosaka T, et al. Expression of snail in upper urinary tract urothelial carcinoma: prognostic significance and implications for tumor invasion. Clin Cancer Res. 2010;16(23):5814-5823.

25. Tanaka N, et al. Acquired platinum resistance enhances tumour angiogenesis through angiotensin II type 1 receptor in bladder cancer. Br J Cancer. 2011;105(9):1331-1337.

26. Bodine SC, et al. Identification of ubiquitin ligases required for skeletal muscle atrophy. Science. 2001;294(5547):1704-1708.

27. Gomes MD, Lecker SH, Jagoe RT, Navon A, Goldberg AL. Atrogin-1, a muscle-specific F-box protein highly expressed during muscle atrophy. Proc Natl Acad Sci U S A. 2001;98(25):14440-14445.

28. Li HH, et al. Atrogin-1/muscle atrophy F-box inhibits calcineurin-dependent cardiac hypertrophy by participating in an SCF ubiquitin ligase complex. J Clin Invest. 2004;114(8):1058-1071.

29. Sandri M, et al. Foxo transcription factors induce the atrophy-related ubiquitin ligase atrogin-1 and cause skeletal muscle atrophy. Cell. 2004;117(3):399-412.

30. Lagirand-Cantaloube J, et al. The initiation factor eIF3-f is a major target for atrogin1/MAFbx function in skeletal muscle atrophy. EMBO J. 2008;27(8):1266-1276.

31. Foletta VC, White LJ, Larsen AE, Léger B, Russell AP. The role and regulation of MAFbx/atrogin-1 and MuRF1 in skeletal muscle atrophy. Pflugers Arch. 2011;461(3):325-335.

32. Zhao P, et al. Slug is a novel downstream target of MyoD. Temporal profiling in muscle regeneration. J Biol Chem. 2002;277(33):30091-30101.

33. Tintignac LA, Lagirand J, Batonnet S, Sirri V, Leibovitch MP, Leibovitch SA. Degradation of MyoD mediated by the SCF (MAFbx) ubiquitin ligase. J Biol Chem. 2005;280(4):2847-2856.

34. Lokireddy S, Wijesoma IW, Sze SK, McFarlane C, Kambadur R, Sharma M. Identification of atrogin-1-targeted proteins during the myostatin-induced skeletal muscle wasting. Am J Physiol, Cell Physiol. 2012;303(5):C512-C529.

35. Ishimoto T, et al. CD44 variant regulates redox status in cancer cells by stabilizing the $\mathrm{xCT}$ subunit of system xc(-) and thereby promotes tumor growth. Cancer Cell. 2011;19(3):387-400.

36. Yamamoto $\mathrm{T}$, et al. Reduced methylation of PFKFB3 in cancer cells shunts glucose towards the pentose phosphate pathway Nat Commun. 2014;5:3480.

37. Tan J, et al. Pharmacologic disruption of Polycomb-repressive complex 2-mediated gene repression selectively induces apoptosis in cancer cells. Genes Dev. 2007;21(9):1050-1063.

38. Chou JL, et al. Promoter hypermethylation of FBXO32, a novel TGF-beta/SMAD4 target gene and tumor suppressor, is associated with poor prognosis in human ovarian cancer. Lab Invest. 2010;90(3):414-425.

39. Guo W, et al. Aberrant methylation and decreased expression of the TGF- $\beta$ /Smad target gene FBXO32 in esophageal squamous cell carcinoma. Cancer. 2014;120(16):2412-2423.

40. Fiskus W, et al. Combined epigenetic therapy with the histone methyltransferase EZH2 inhibitor 3-deazaneplanocin A and the histone deacetylase inhibitor panobinostat against human AML cells. Blood. 2009;114(13):2733-2743.

41. Fiskus W, et al. Superior efficacy of a combined epigenetic therapy against human mantle cell lymphoma cells. Clin Cancer Res. 2012;18(22):6227-6238.

42. Julie LC, Sabrina BP, Marie-Pierre L, Leibovitch SA. Identification of essential sequences for cellular localization in the musclespecific ubiquitin E3 ligase MAFbx/Atrogin 1. FEBS Lett. 2012;586(4):362-367.

43. Jamieson ER, Lippard SJ. Structure, recognition, and processing of cisplatin-DNA adducts. Chem Rev. 1999;99(9):2467-2498.

44. Xie M, et al. Activation of Notch-1 enhances epithelial-mesenchymal transition in gefitinib-acquired resistant lung cancer cells. J Cell Biochem. 2012;113(5):1501-1513.

45. Shien K, et al. Acquired resistance to EGFR inhibitors is associated with a manifestation of stem cell-like properties in cancer cells. Cancer Res. 2013;73(10):3051-3061. 